\title{
Still a Long Way to Fully Understanding the Molecular Mechanism of Escherichia coli Purine Nucleoside Phosphorylase ${ }^{\dagger}$
}

\author{
Zoran Štefanić, ${ }^{a}$ Goran Mikleušević, ${ }^{a}$ Marta Narczyk, ${ }^{b}$ Beata Wielgus-Kutrowska, ${ }^{\text {b }}$ \\ Agnieszka Bzowska, ${ }^{b}$ and Marija Luić ${ }^{a, *}$ \\ ${ }^{a}$ Division of Physical Chemistry, Ruđer Bošković Institute, P.O. Box 180, HR-10002 Zagreb, Croatia \\ ${ }^{\mathrm{b}}$ Division of Biophysics, Institute of Experimental Physics, University of Warsaw, \\ Zwirki i Wigury 93, 02-089 Warsaw, Poland
}

RECEIVED JUNE 15, 2012; REVISED DECEMBER 14, 2012; ACCEPTED JANUARY 10, 2013

\begin{abstract}
The results of several decades of studying the catalytic mechanism of Escherichia coli purine nucleoside phosphorylases (PNP) by solution studies and crystal structure determinations are presented. Potentially PNPs can be used for enzyme-activating prodrug gene therapy against solid tumours because of the differences in specificity between human and E. coli PNPs. Biologically active form of PNP from $E$. coli is a homohexamer that catalyses the phosphorolytic cleavage of the glycosidic bond of purine nucleosides. Two conformations of the active site are possible after substrate(s) binding: open and closed. A series of determined 3D-structures of PNP binary and ternary complexes facilitated the prediction of the main steps in the catalytic mechanism. For their validation the active site mutants: Arg24Ala, Asp204Ala, Arg217Ala, Asp204Asn and double mutant Asp204Ala/Arg217Ala were prepared. The activity tests confirm that catalysis involves protonation of the purine base at position N7 and give better insight into the cooperativity between subunits in this oligomeric enzyme. (doi: 10.5562/cca2116)
\end{abstract}

Keywords: purine nucleoside phosphorylase, catalysis, cooperativity

\section{INTRODUCTION}

Catalysis is an important process in chemistry and enzymology. Enzymes play a key role in most biological processes because of their ability to accelerate a variety of reactions by many orders of magnitude thus making them feasible at biologically relevant times. For this reason the broad interest in understanding of the catalytic power of enzymes on the atomic level is not surprising. Oligomeric proteins might be especially challenging in this respect since their functioning might be additionally complicated by communication i.e. cooperativity between subunits.

The role of protein structure in enzyme catalysis is well established and the conservation of structural features provides vital clues to their part in function. Recently, it has been proposed that the protein function may involve multiple conformations and that flexibility may be closely linked to protein function, including enzyme catalysis.

Purine nucleoside phosphorylases (PNP) play a key role in the purine salvage pathway. They catalyse the phosphorolytic cleavage of the glycosidic bond of purine (2'-deoxy)nucleosides, generating the corresponding free base and (2'-deoxy)ribose 1-phosphate. Two classes of PNPs have been identified: homotrimers (low-molecular mass PNPs) specific for 6-oxopurines and homohexamers (high-molecular mass PNPs) that accept both 6-oxopurines and 6-aminopurines. Homotrimers are found mostly in mammals and in only a few bacteria (for example Bacillus subtilis and Cellulomonas $s p$.), while homohexamers are characteristic for most bacteria. ${ }^{1}$ Differences in specificity between these two groups of enzymes, low- and high-mass PNPs, can be used for enzyme-activating prodrug gene therapy enabling selective killing of tumour cells expressing the Escherichia coli PNP gene. ${ }^{2,3,4}$ Recently, targeted expression of E. coli PNP, as a novel suicide gene in the prostate cancer, in combination with fludarabine induced a massive prostate's tumour cell death and prolonged life span without systemic cytotoxicity, ${ }^{5}$ confirming application possibilities for this enzyme. In this review we present our results of long-range studying of the catalytic mechanism of $E$. coli PNP. In these studies we have used different methods: kinetic and binding studies in solution (using steady-state kinetic measurements,

\footnotetext{
$\dagger$ This article belongs to the Special Issue devoted to the $85^{\text {th }}$ anniversary of Croatica Chemica Acta.

* Author to whom correspondence should be addressed. (E-mail: marija.luic@irb.hr)
} 

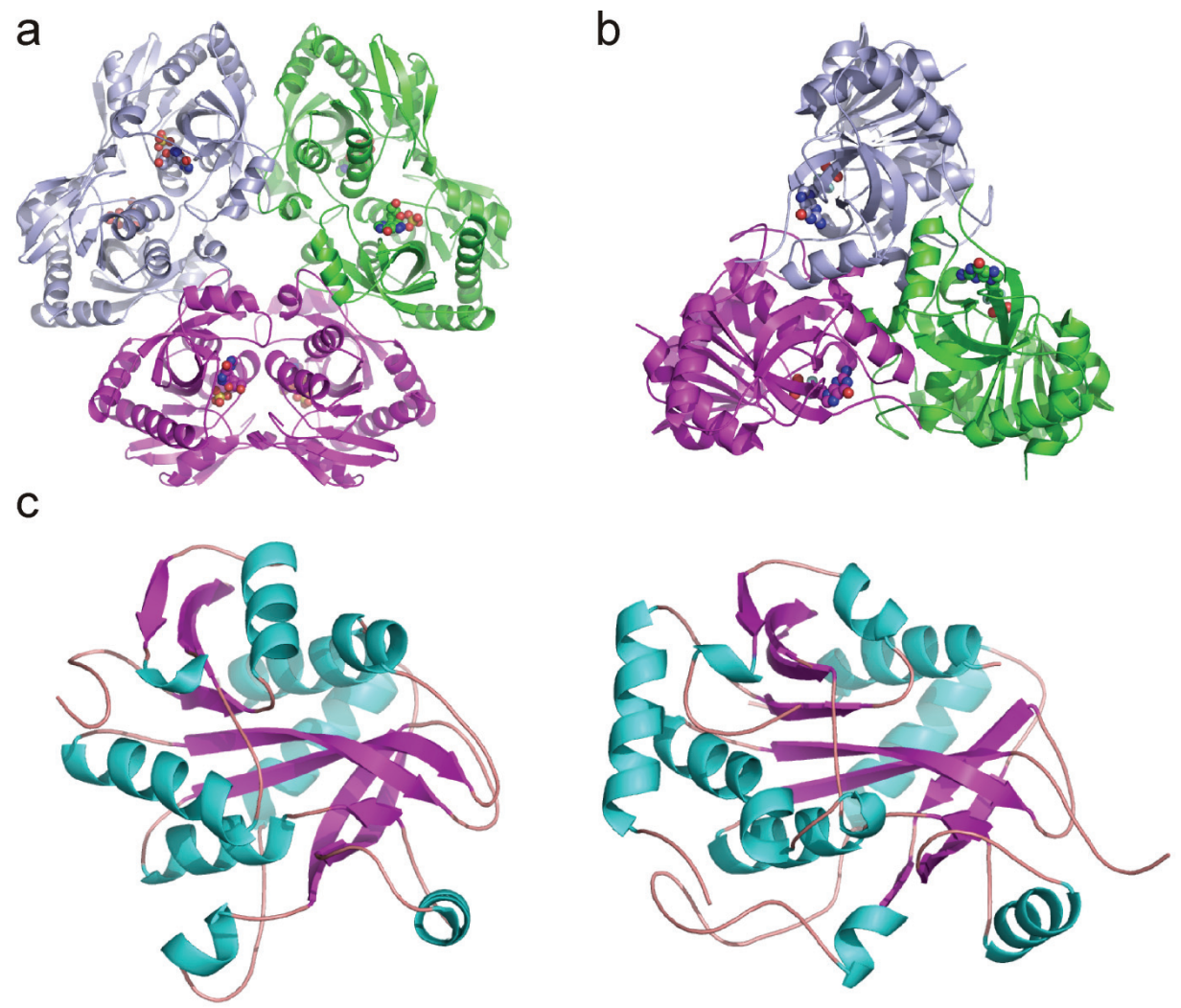

Figure 1. Comparison of E. coli PNP complexed with formycin B and phosphate/sulphate (PDB code 1A69) and calf spleen PNP complexed with 9-(5,5-difluoro-5-phosphonopenthyl)guanine (PDB code 1V48). Atoms of ligands bound in the active sites are shown as spheres. PNP from E. coli is a hexamer and can be viewed as the trimer of dimers shown in blue, magenta and green (a), while PNP from calf spleen forms a trimer (b). Despite the difference of oligomeric state and no amino-acid sequence homology the overall structure of monomers of both enzymes from E. coli (left) and calf spleen (right) is very similar (c).

stopped-flow approach, fluorimetric titrations and isothermal titration calorimetry), X-ray crystallography and site-directed mutagenesis. A series of determined 3Dstructures of PNP binary and ternary complexes enabled us to predict the main steps in the catalytic mechanism and to identify conformational changes resulting in negative cooperativity between monomers in a dimer during phosphate binding initially predicted by solution studies. Unfortunately, the obtained results provide no conclusion about communication between dimers in biologically active hexamers. Namely, based on the current knowledge the minimum catalytic unit could be a dimer but since the enzyme's active form is a hexamer probably also some dimer-dimer communication exists. Recently, an unexpected phosphate binding mode has been revealed. ${ }^{6}$ Obviously, new methods and new extensive studies are needed to fully understand cooperativity of this allosteric protein.

\section{FIRST CRYSTAL STRUCTURE OF E. coli PNP}

In 1997 . the first $E$. coli PNP crystal structure solved at $2.0 \AA$ resolution (PDB code 1ECP) was published by
Mao and coworkers. ${ }^{7}$ The crystals of the unliganded enzyme were obtained from crystallization solutions containing $12-14 \%(w / v)$ PEG 4000 with 50 mmol $\mathrm{dm}^{-3}$ citrate buffer, $\mathrm{pH}=4.8-5.0$. They belong to the monoclinic space group $P 2{ }_{1}$ with cell parameters $a=$ $89.5, b=111.5$ and $c=74.8 \AA, \beta=110.7^{\circ}$ and one hexamer per asymmetric unit. This structure revealed that $E$. coli $\mathrm{PNP}$ is a cylinder-shaped hexamer with $\mathrm{D}_{3}$ symmetry whose diameter is about $100 \AA$ and thickness about $60 \AA$ (Figure 1a).

In the centre of the hexamer there is a channel of approximately $20 \AA$ in diameter. For the first time it was shown that the overall structure of the hexamer can be better described as a trimer of dimers and that each dimer has two complete active sites that are face to face oriented. Active site of one monomer in a dimer is completed by two amino acids from the adjacent monomer. These two amino acids are Arg43 and His4. It was predicted, although no ligands were present in the active site, that Arg43 from one monomer participates in binding of the phosphate from another monomer by both, electrostatic interactions and hydrogen bonding and that His 4 probably forms one or two hydrogen bonds with the hydroxyl group of the ribose. 


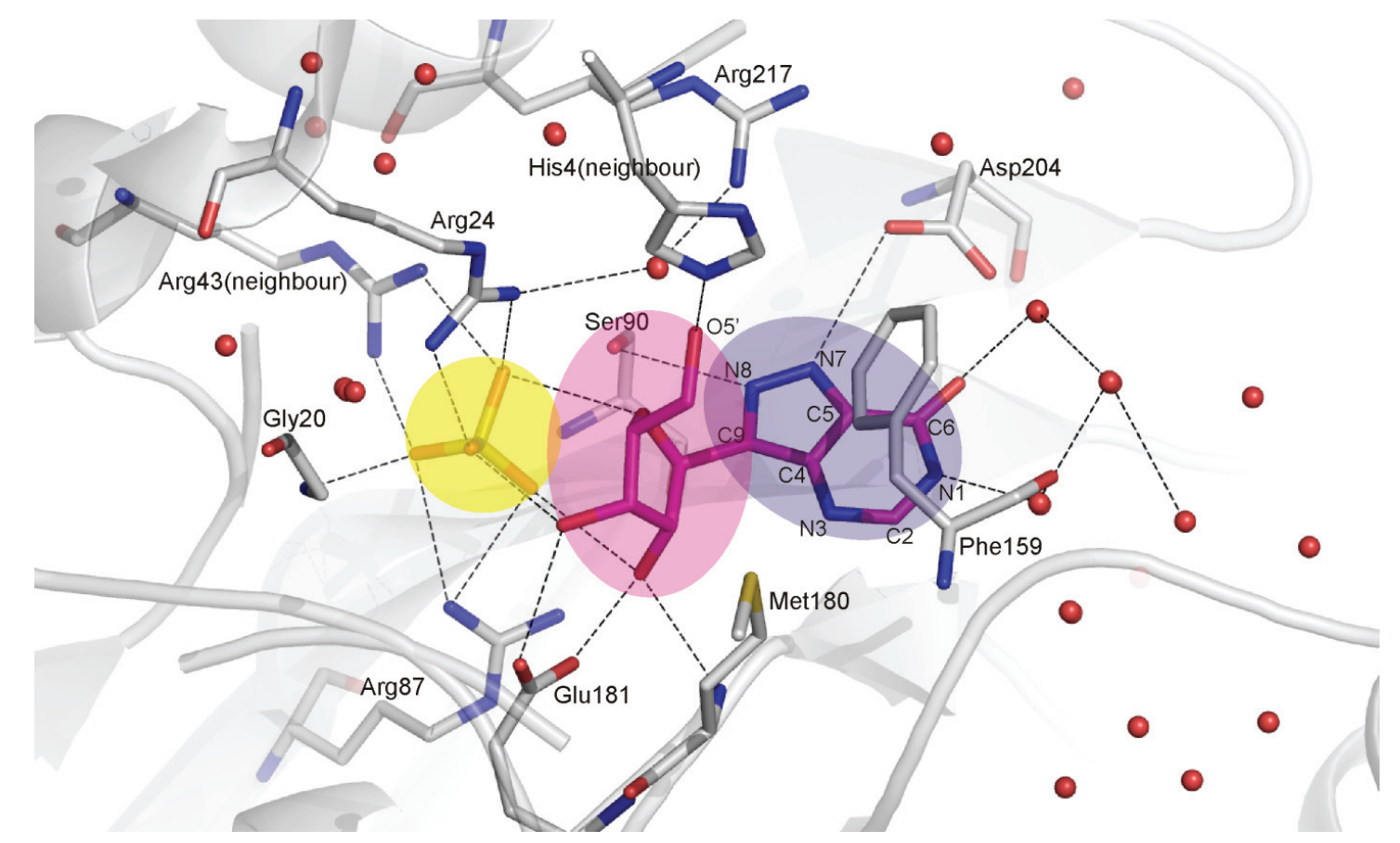

Figure 2. Active site of PNP from E. coli with formycin B and sulphate ion bound in the active site. Shaded areas represent the binding sites for sulphate, pentose and purine base. Major hydrogen bonds are depicted as dashed lines and water molecules are shown as red spheres.

Each monomer consists of a central nine-stranded mixed $\beta$-sheet classified as a mixed $\beta$-barrel surrounded by eight $\alpha$-helices (Figure 1c). Despite low sequence identity between $E$. coli and mammalian purine nucleoside phosphorylases, ${ }^{8}$ similar arrangement of the central $\beta$-sheet surrounded with $\alpha$-helices was found in both enzymes (Figure 1c). By superimposing the structures of these two monomers it could be seen that the central $\beta$-sheet is structurally conserved. Additionally, comparison of their secondary structure elements reveals that all nine $\beta$-strands as well as some helices consist of almost the same number of residues. ${ }^{9}$ In $E$. coli PNP the $\beta$ strand content is about $27 \%$ and in human PNP $23 \%$. The $\alpha$-helical content is $34 \%$ in E. coli compared to $33 \%$ in human enzyme. This means that $E$. coli PNP has $5 \%$ more secondary structure elements than human even though the E. coli PNP monomer has 238 and human PNP molecule 289 amino acids. Proportionally, human PNP trimer has more loops and turns. In hexameric $E$. coli PNP loops and turns are of similar length while in mammalian PNPs they are significantly different. The variation in trimeric and hexameric active sites comes from differences in loops and turns.

In addition to the native protein, Mao and coworkers have also prepared complexes with sulphate, ribose-1-phosphate and 6-iodopurine, crystallized them and examined their structures. Although all small molecules were visible in electron density maps, their accurate location in the active site was not possible. ${ }^{7}$
Comparison of E. coli PNP with the 3D-structure of calf spleen enzyme ${ }^{10}$ shows that the active centres of both enzymes are located in the same region of the monomer, and in the same orientation relative to the central $\beta$-barrel (Figure 1a and b).

\section{TERNARY COMPLEX OF $E$. coli PNP WITH FORMYCIN B AND PHOSPHATE/SULPHATE}

One year later, in the year 1998, a crystal structure of the ternary complex of $E$. coli PNP with formycin B (FB) and phosphate/sulphate at $2.1 \AA$ resolution (PDB code 1A69) was published by Koellner et al. ${ }^{9}$ Formycin B is an antibiotic which is a structural analogue of the substrate inosine. Formycins have 8-aza-9-deazapurine instead of purine bases and the glycosidic $\mathrm{C}-\mathrm{N}$ bond between base and sugar moiety present in PNP natural substrates is replaced by $\mathrm{C}-\mathrm{C}$ bond that can not be cleaved by the enzyme. Consequently, formycins are PNP inhibitors. The complex crystallized from $50 \mathrm{mmol} \mathrm{dm}{ }^{-3}$ citric buffer, $\mathrm{pH}=5.2-5.4$, and $28-35 \%$ ammonium sulphate $(w / v)$ in hexagonal space group $P 6_{1} 22$ with cell dimensions $a=$ 123.11, $c=241.22 \AA$ and with one half of the hexamer in the asymmetric unit. The biologically active hexamer is formed through crystallographic 2-fold axes and, as in previously described structure, represents a trimer of dimers. In this structure the ligands bound in the active sites were very well visible in the electron density maps and their positions could be well located. 


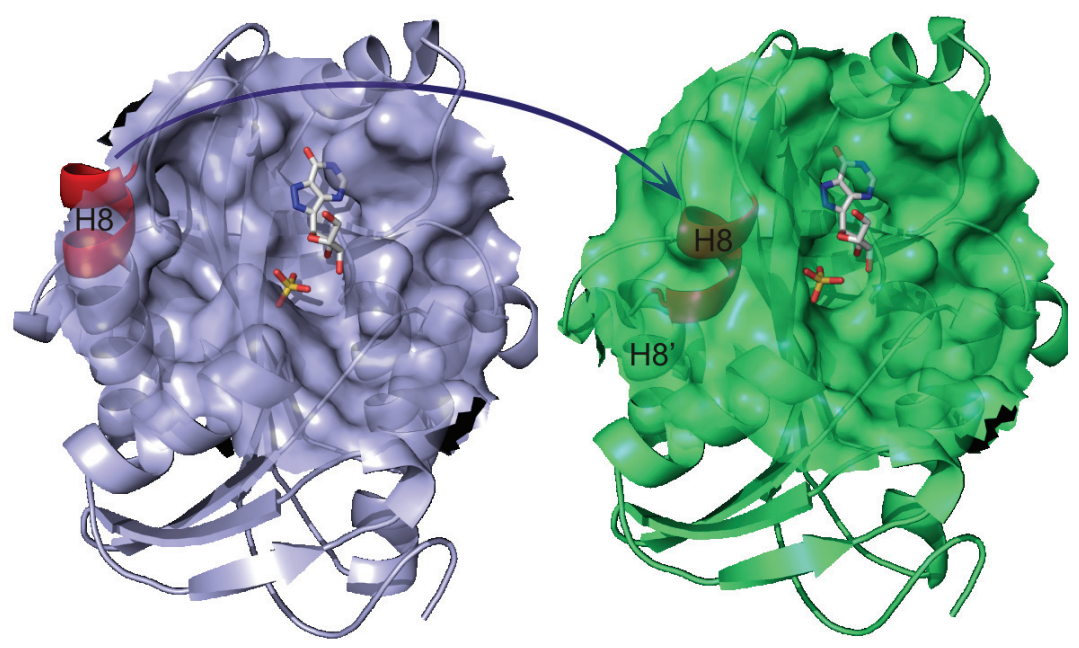

Figure 3. Partial closing of the active site pocket resulting from the conformational change in the helix H8 from elongated (left) to segmented (right) configuration leading from open (left) to closed (right) conformation of the E. coli PNP active site (PDB code 1A69).

The active sites of the monomers comprising a dimer are located one "above" and the other "below" the plane formed by the centre of gravity of the monomers and the distance between them is about $19 \AA$. It can be divided into three parts: the phosphate, the ribose and the base binding site (Figure 2).

The phosphate binding site is occupied either by sulphate ion present in the crystallization solution or by phosphate ion present in solutions used during the purification procedure. All our efforts to crystallize the complex without ammonium sulphate as a precipitating agent failed at that time (see below). Sulphate ion is a weak inhibitor of $E$. coli PNP $\left(K_{\mathrm{i}}=1.2 \mathrm{mmol} \mathrm{dm}^{-3}\right){ }^{11}$ Phosphate binding region is the most buried part of the E. coli PNP active site and consists of Gly $20 \mathrm{~N}$, $\operatorname{Arg} 24 \mathrm{~N}^{\eta}, \operatorname{Arg} 87 \mathrm{~N}^{\eta}, \operatorname{Ser} 90 \mathrm{~N}$, Ser90 $\mathrm{O}^{\gamma}$ and $\operatorname{Arg} 43 \mathrm{~N}^{\eta}$ from the adjacent monomer. The three arginine residues make the phosphate active site positively charged. All phosphate/sulphate oxygen atoms make at least two hydrogen bonds or salt bridges with the protein. This could be the reason for the high affinity of phosphate for E. coli PNP. ${ }^{11}$

The ribose binding site is very close to the position of the phosphate/sulphate ion and all sugar oxygen atoms have direct contact with it, except for one, O5', which forms a hydrogen bond with His4 $\mathrm{N}^{\varepsilon}$ from the neighbouring monomer. Ribose oxygen atoms make several direct contacts to the protein and some bridged via water molecules.

The base binding site is exposed to the solvent. There is a network of water molecules from the inhibitor atoms to the bulk water molecules. Additionally, several interactions between the base, Phe159 and Tyr160, reminiscent of the "aromatic-aromatic" interactions in protein contribute to the stabilization of tertiary and quaternary protein structure. ${ }^{12}$

\section{TERNARY COMPLEX OF E. coli PNP WITH FORMYCIN A DERIVATIVES AND PHOS- PHATE/SULPHATE - HYPOTHESIS OF CATA- LYTIC MECHANISM}

The crystal structure of the ternary complex of E. coli PNP with formycin A derivatives and phosphate/ sulphate at $2.0 \AA$ resolution (PDB code $1 \mathrm{~K} 9 \mathrm{~S}$ ) was published by Koellner et al. in 2002. ${ }^{13}$ The idea was to crystallize the PNP complex with the 6-methylformycin A known as the most potent $E$. coli PNP inhibitor. ${ }^{14}$ Unfortunately, formycins occur in solution as a mixture of two tautomeric forms. ${ }^{15} \mathrm{Ad}-$ ditionally, 6-methylformycin $\mathrm{A}$ in water solution undergoes rearrangement to four formycin derivatives. One of them, N7-methylformycin A, was found in three of six active sites of the crystal structure of the ternary complex. The complex crystallized from the same crystallization condition as a complex with formycin B: $50 \mathrm{mmol} \mathrm{dm}^{-3}$ citric buffer, $\mathrm{pH}=5.2-5.4$, and $28-35 \%$ ammonium sulphate $(w / v)$. But this time two different crystal forms appeared: hexagonal rods belonging to the already described hexagonal space group $P 6_{1} 22$ with cell dimensions $a=123.11, c=241.22 \AA$ and one half of the hexamer in the asymmetric unit and tetragonal bypiramids belonging to the $P 4_{1} 2_{1} 2$ space group with cell dimensions $a=b=178.8, c=167.9 \AA$ and a whole hexamer in the asymmetric unit. Having the whole hexamer in the asymmetric unit is very convenient since in this case no bias due to crystallographic symmetry is possible and therefore a tetragonal form was chosen for the data collection.

The overall structure is very similar to the structures described in previous structural studies, but here, for the first time, two clearly different conformations of the active site were observed (Figure 3). 
1)

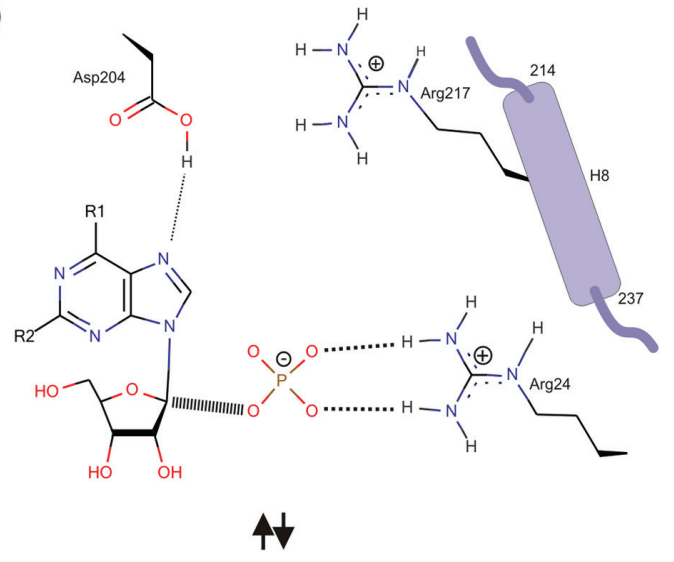

2)

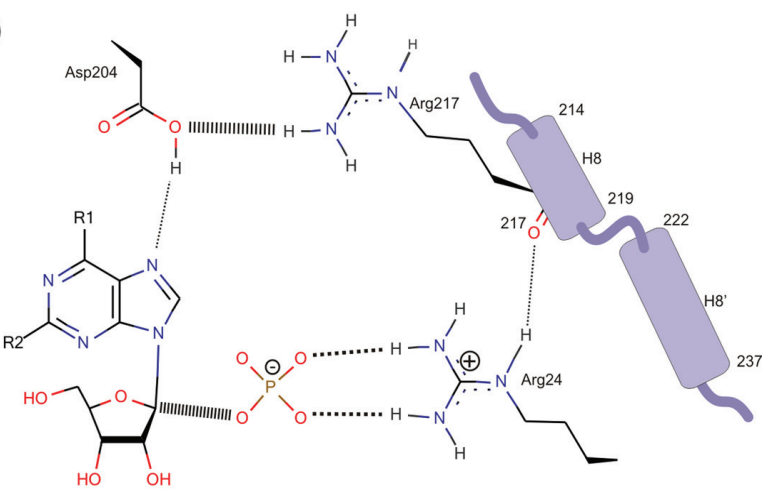

$4 \downarrow$

3)

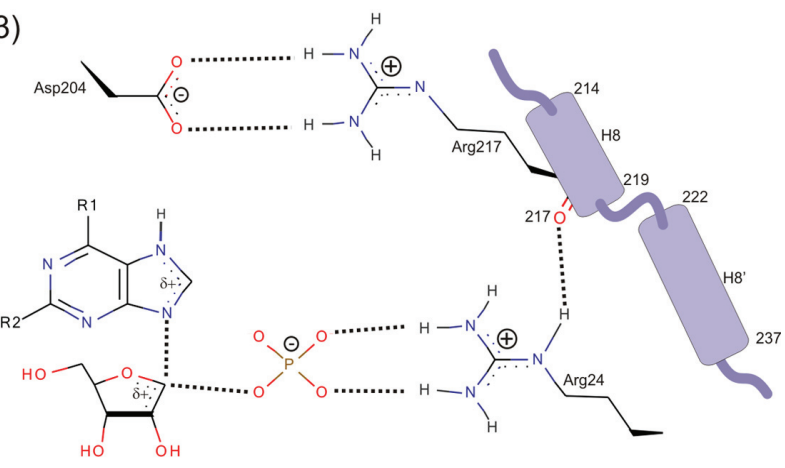

Figure 4. Schematic presentation of the proposed catalytic mechanism of E. coli purine nucleoside phosphorylase. Asp204 must be in the protonated form prior nucleoside binding. (1) loose-binding conformation of $E$. coli PNP with continuous $\alpha$ helix $\mathrm{H} 8$ and hydrogen bond donation of Asp204 side-chain to the base at position N7. The side-chain Arg24 is floppy. (2) Conformational change occurs that results in breaking of $\alpha$ helix $\mathrm{H} 8$ into two segments. Arg217 side-chain moves closer to Asp204. Binding of phosphate anion stabilise Arg24. (3) Positively charged transition state is formed by proton transfer from side-chain Asp204 to N7 position of base followed by formation of a salt bridge between Arg217 and Asp204.
This difference stems from a flexible helix $\mathrm{H} 8$ comprising amino acid residues from 214 to 236 . In one conformation this helix is continuous and in the other one is segmented. The conformer with a continuous $\mathrm{H} 8$ has the entry into the active site wide open and the ligands are probably bound only loosely. This conformation is called "open" or "loose binding" conformation. In the conformer with the segmented $\mathrm{H} 8$ we can distinguish smaller helix comprising residues 214 do 219 called H8 separated by a $\gamma$-turn from bigger helix (223-236) called H8'. The entry into the active centre of this conformer is partially closed by a smaller helix $\mathrm{H} 8$ and the ligands are probably bound much more tightly. This conformation is called "closed" or "tight binding" conformation. Each dimer in a hexamer observed in this structure has one monomer in open and one in closed conformation. The temperature factors of the atoms at the N-terminus of the continuous $\mathrm{H} 8$ helix are high indicating its flexibility. Actually, already in the ternary complex with the formycin B and phosphate/sulphate ion these two conformations were noticed, but due to crystal symmetry and disorder (existence of highly flexible regions), an unequivocal interpretation of the electron density maps was not possible.

In the monomer with the active site in closed conformation one phosphate/sulphate ion and one 6-methylformycin A molecule are bound, as was expected. Something unexpected occurred in the monomer with the active site in open conformation. Namely, in this case the inhibitor molecule could not be modelled as 6-methylformycin A. A detailed inspection of the difference electron density maps indicated that $\mathrm{N} 7$-methylformicin A, a main water hydrolysis product of 6-methylformycin A, fits much better into it. We supposed that initially 6-methylformycin A was bound to all six active sites and that in the course of time between setting up the crystallization experiment and data collection (3 months) it has undergone hydrolysis to N7-methylformicin A but only in three monomers with the active sites in the open conformation. This observation was also taken into account in formulating the hypothesis that open conformation binds ligands loosely, while the closed conformation is responsible for a more strong binding.

Studies of phosphate binding to E. coli PNP in solution revealed that there are two dissociation constants, $29.4 \mu \mathrm{mol} \mathrm{dm}{ }^{-3}$ and $1.12 \mathrm{mmol} \mathrm{dm}{ }^{-3}$, while sulphate binding, as already reported, is described by only one dissociation constant ${ }^{11}, 1.36 \mathrm{mmol} \mathrm{dm}^{-3}$. Protein was in contact with phosphate during purification and the crystals for data collection were obtained from the ammonium sulphate solution. Since phosphate and sulphate ions can not be distinguished by X-ray diffraction data at the given resolution, we have presumed that sulphate ions 
are bound in the open active sites (second $K_{\mathrm{d}}$ for phosphate and $K_{\mathrm{d}}$ for sulphate are similar and the crystals grew from excess of sulphate), while it is not clear whether sulphate replaced phosphate in the closed active sites. Solution studies have shown potent binding of 6-methylformycin A only in the presence of phosphate which indicates that probably phosphate and not sulphate is bound in the closed active sites.

It was suggested that in $E$. coli PNP catalytic mechanism the purine base is protonated by a suitable acid at $\mathrm{N} 7^{16,17}$. The base binding modes observed in the ternary complex of $E$. coli PNP with formycin A derivatives and phosphate/sulphate ions give us more details about the catalytic mechanism of this enzyme. Although formycins are not natural substrates, we can expect that in the natural substrates like in formycins, N7 atom is at the hydrogen bond distance to Asp204 $\mathrm{O}^{\gamma}$. This amino acid is conserved in all PNPs homologues with E. coli $\mathrm{PNP}^{1}$. If Asp204 is protonated in native protein, it could be that this acid donates a proton to bound nucleoside. In the closed conformation of the active site Asp204O $\delta^{1}$ and $\operatorname{Asp} 204 \mathrm{O} \delta^{2}$ accept two hydrogen bonds from Arg217 implying that the side chain is charged. In the case of Asp204 protonation, the proton transfer from Asp204 O $\delta-\mathrm{H}$ triggered by the conformational change from open to close binding site could be supposed.

According to the above described observations we proposed a catalytic mechanism for $E$. coli $\mathrm{PNP}^{13}$ and illustrated it in the Figure 4.

The catalysis proceeds in three steps. (1) In the open conformation of the apo enzyme helix H8 is continuous, Arg217 is away from Asp204 which is in acidic form. Both, phosphate and nucleoside can enter the active site, however solution studies show that nucleoside binds much stronger, if not exclusively, to the enzyme/phosphate complex. ${ }^{13,18}$ The neutral side-chain of Asp204 donates a hydrogen bond to the purine nitrogen N7. (2) Phosphate binding stabilizes flexible side chain of Arg24 and the conditions for segmentation of $\mathrm{H} 8$ are formed. After segmentation of $\mathrm{H} 8$ the active site is in closed conformation. Since the entrance to the active site pocket is only partially closed (Figure 3 ), it is possible that nucleoside enters without additional changes. The other possibility for nucleoside to enter the active site is its re-opening. (3) Due to the described conformational change the guanidinium group of Arg217 comes in contact with the acid group of Asp204. Proton transfer to the base occurs followed by formation of a salt bridge between Arg217 and Asp204. The positively charged purine base leads to the ribooxocarbenium ion character of the ribose which represents the transition state of the glycosidic bond cleavage in acid hydrolysis, first proposed for trimericPNPs by Kline and Schramm in $1995 .{ }^{19}$ The key reaction between the enzyme and the substrate is at the position N7 of the base. This is consistent with a broad specificity of $E$. coli PNP which accepts both 6-oxo and 6-aminopurine nucleosides while trimeric PNPs accept only 6-aminopurines as substrates.

Determination of this crystal structure was a significant step forward in elucidating the molecular mechanism of E. coli PNP. Together with the solution studies it showed that two monomers in the dimer (regarded as a catalytic unit) are not independent and that probably only one at the time can be in the closed conformation. It offered a possible explanation for the observed negative cooperativity between monomers in a dimer by phosphate binding to the enzyme. ${ }^{11}$ Alternate arrangement of monomers with open and closed conformations in a hexamer implies that there must exist information transfer also between dimers in a hexamer. How they communicate remains to be elucidated. Crystal structure does not offer any clue to answer this question.

\section{VALIDATION OF THE PROPOSED MECHA- NISM}

In order to validate the proposed molecular mechanism of PNP and to answer many open questions regarding the PNP-catalysed reaction we accomplished additional studies including site-directed mutagenesis, kinetic and binding measurements and determination of new crystal structures. The role of particular catalytic amino acid was studied by preparing active site mutants: Arg24Ala, Asp204Ala, Arg217Ala, Asp204Asn and double mutant Asp204Ala/Arg217Ala. Their activity vs. several natural (inosine, adenosine and guanosine) and 7-methylguanosine substrates was determined (Table 1 - reproduced from G. Mikleušević et al.). ${ }^{20}$ Mutation of catalytic amino acids significantly reduced the activity $v s$. natural substrates, proving the significance of these amino acids for the enzyme activity. Activity of all prepared mutants vs. 7-methylguanosine was either the same or higher than the activity of the wild type enzyme. This supports our assumption that the protonation at position N7 is essential for catalysis. Residues responsible for protonation are Asp204 and Arg217. Mutation of one or both of them considerably lowered the activity $v s$. natural substrates.

7-methylguanosine has the methyl group at position N7 and protonation is not possible. Therefore, mutation of amino acids Asp204 and Arg217 had no influence or even favourable effect on the catalysis. Double mutant Asp204Ala/Arg217Ala shows no lower activity vs. natural substrates than the Asp204Ala mutant indicating the cooperativity action of these two residues in the course of purine base protonation. 
Table 1. Enzymatic activity in U/mg of the active site PNP mutants $v s$. natural substrates (inosine, adenosine and guanosine) and $v$ s. 7-methylguanosine. Data were obtained at $\mathrm{pH}=7.0,50 \mathrm{mmol} \mathrm{dm}^{-3}$ Hepes buffer, $298 \mathrm{~K}$, with $50 \mathrm{mmol} \mathrm{dm}^{-3}$ phosphate. For each substrate activity is also shown in \% vs. wild-type enzyme. One unit of PNP enzymatic activity is defined as the amount of enzyme that causes phosphorolysis of one $\mu \mathrm{mol}$ of the purine nucleoside to the corresponding base and ribose-1-phosphate per min

\begin{tabular}{lcccccccc}
\hline \multirow{2}{*}{ Substrate/Mutant } & \multicolumn{2}{c}{ Ino } & \multicolumn{2}{c}{ Ado } & \multicolumn{2}{c}{ Guo } & \multicolumn{3}{c}{$\mathrm{m}^{7} \mathrm{Guo}$} \\
\cline { 2 - 9 } & $\mathrm{U} / \mathrm{mg}$ & $\%$ & $\mathrm{U} / \mathrm{mg}$ & $\%$ & $\mathrm{U} / \mathrm{mg}$ & $\%$ & $\mathrm{U} / \mathrm{mg}$ & $\%$ \\
\hline Wild-type recombinant & $103.2 \pm 4.0$ & 100 & $58.5 \pm 5.9$ & 100 & $60.4 \pm 6.7$ & 100 & $21.4 \pm 6.1$ & 100 \\
Arg24Ala & $0.18 \pm 0.01$ & 0.2 & $0.36 \pm 0.05$ & 0.6 & $0.09 \pm 0.02$ & 0.2 & $6.4 \pm 0.5$ & 29.9 \\
Asp204Ala & $0.09 \pm 0.01$ & 0.1 & $0.05 \pm 0.05$ & 0.1 & $0.06 \pm 0.01$ & 0.1 & $20.0 \pm 4.6$ & 93.5 \\
Asp204Asn & $1.4 \pm 0.1$ & 1.4 & $0.07 \pm 0.03$ & 0.1 & $2.1 \pm 0.2$ & 3.5 & $6.9 \pm 2.6$ & 32.2 \\
Arg217Ala & $0.41 \pm 0.03$ & 0.4 & $6.3 \pm 1.3$ & 10.8 & $0.36 \pm 0.04$ & 0.6 & $21.3 \pm 0.8$ & 99.5 \\
Asp204Ala/Arg217Ala & $0.14 \pm 0.02$ & 0.1 & $0.13 \pm 0.01$ & 0.2 & $0.07 \pm 0.02$ & 0.1 & $46.9 \pm 5.2$ & 219.2 \\
\hline
\end{tabular}

Arg24Ala mutant shows very low activity vs. all studied natural substrates which proves its importance in the reaction mechanism as well the necessity of protonation of the purine base at position N7.

\section{BINARY COMPLEXES OF WILD TYPE AND Arg24Ala MUTANT WITH PHOSPHATE/- SULPHATE - NEW ARRANGEMENT OF OPEN AND CLOSED ACTIVE SITE IN HEXAMERS}

Three binary complexes of the wild type enzyme (with phosphate, with phosphate or sulphate and with sulphate) and one of the Arg24Ala mutant with phosphate or sulphate were prepared, crystallized and their crystal structures determined. ${ }^{20}$ Although grown from very similar conditions, they crystallized in four different space groups. Hexamers are formed from symmetrically independent monomers in all structures except for the wild type binary complex with sulphate where hexamer is formed by crystallographic two-fold axes operating on the three symmetrically independent monomers. Hence, only in the structure of the wild type binary complex with sulphate differences between monomers forming the dimer could be biased by crystallographic symmetry. In all the structures phosphate binding sites are occupied by phosphate and/or sulphate ions.

As expected, in the crystal structures of two binary complexes, that of wild type with sulphate and of the Arg24Ala mutant with phosphate/sulphate ions only open conformation of the active sites was observed. These structures confirmed our previously reported observation that conformational change is the result of the phosphate binding (phosphate is probably the first substrate that binds to the enzyme) and its interaction with Arg24. No conformational change in the binary complex with sulphate is consistent with the fact that sulphate is actually an inhibitor and not a substrate. In the structure of Arg24Ala mutant no interaction between phosphate and Arg24 was possible, hence binding of sulphate can not cause the conformational change.

The most important structure for our studies from this series was the structure of the binary complex of the wild type with phosphate obtained from a crystal grown in the crystallization conditions without ammonium sulphate. The structure was refined at $2.0 \AA$ resolution (PDB code $3 \mathrm{OOH}$ ). This was the first time that we have got crystals in the absence of sulphate. Only on the basis of this structure non-biased discussion about the role of phosphate in conformational change of the active site is possible. The complex crystallizes in the $P 2_{1}$ space group with as many as three hexamers in the asymmetric unit. Hexamers stack on top of each other along the $c$ crystallographic axes. To our surprise, instead of three monomers in the open and three in the closed conformation, each of three hexamers in the asymmetric unit had two active sites in closed and four in open conformation. Two closed active sites are next to each other in all three hexamers but belong to neighbouring dimers (Figure 5).

Higher flexibility of the open active site conformations can be seen from higher displacement parameters of their residues compared with those in the closed active site conformation. Detailed inspection of the active sites showed also higher flexibility of the Arg24 in the open than in the closed conformation of active sites. In all closed active sites Arg24 is hydrogenbonded to phosphate while in most, but not all, open active sites Arg24 points away from phosphate. The same is true for the bound phosphate ions; the mean displacement parameter of phosphates in open and closed conformations is 39 and $18.8 \AA^{2}$, respectively. Higher thermal mobility of phosphate ions in the open conformation is observed also in both of the above described PNP ternary complexes. 

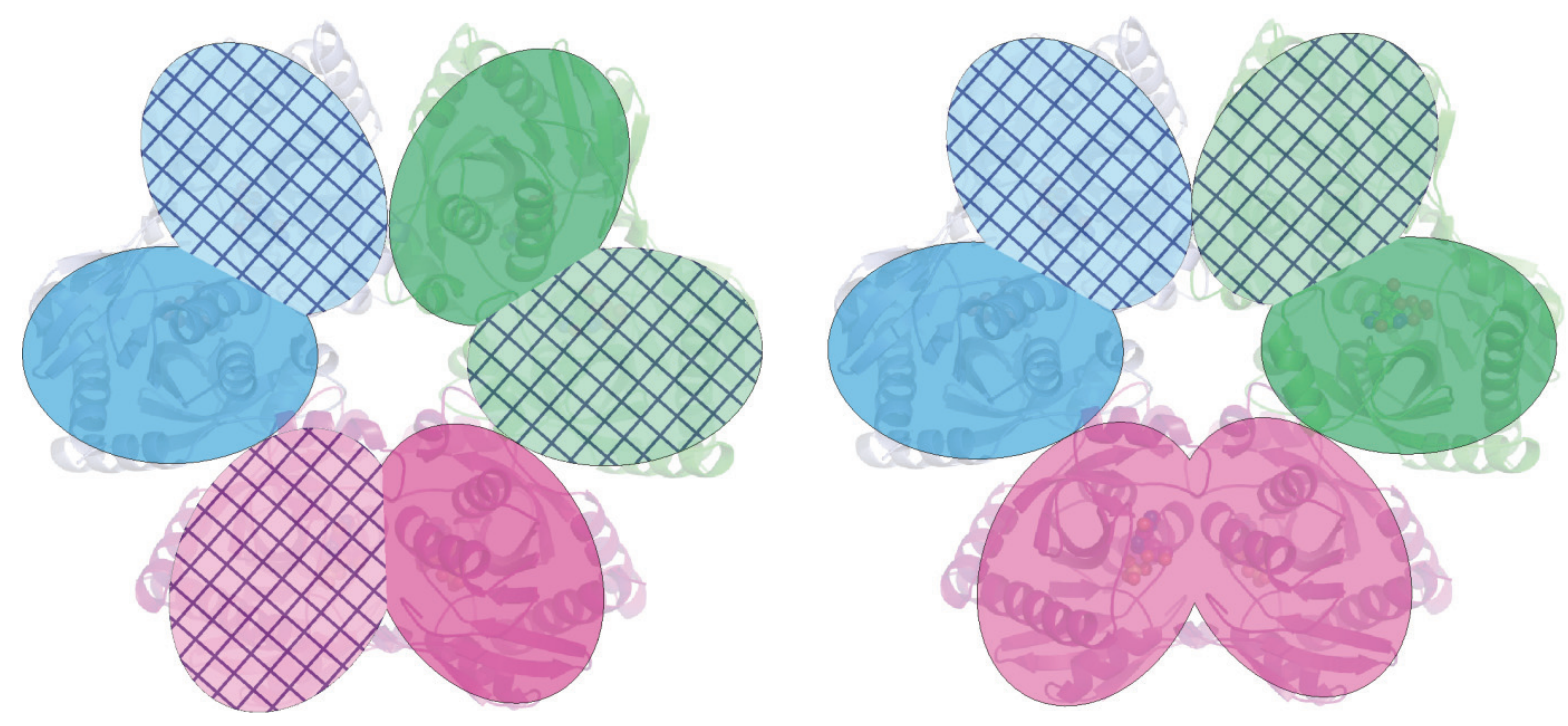

Figure 5. The arrangement of monomers with open and closed active sites in PNP complexed with formycin A derivatives and phosphate/sulphate (PDB code 1K9S) and in binary complex with phosphate (PDB code 3OOH). In the former the monomers alternate regularly ("3+3" arrangement) where each dimer contains one active site in open and one in closed conformation while the later makes ("4+2" arrangement) with two active sites in closed conformation next to each other. Dimers are represented by the same colour, monomers with open active sites by clear ovals and monomers with closed active sites as crosshatched ovals.

In order to prepare a binary complex with only one half of the active sites occupied by phosphate ions (three active sites supposed to be in closed conformation), we used the concentration of phosphate in the range between the first and second dissociation constant of the PNP-phosphate complex, $29.4 \mu \mathrm{mol} \mathrm{dm}{ }^{-3}$ and $1.12 \mathrm{mmol} \mathrm{dm}^{-3}$. For the crystallization experiments we used crystallization solutions with ammonium sulphate (the best known precipitant for E. coli PNP), and PEG, hoping to get phosphate binding sites in open conformation free of any ligand. Unfortunately, crystals appeared only in ammonium sulphate crystallization conditions. They belong to an orthorhombic $P 2_{1} 2_{1} 2_{1}$ space group with one hexamer in the asymmetric unit. The structure was refined at $2.9 \AA$ resolution (PDB code 3OOE). As we expected, all phosphate binding sites in this hexamer were occupied. Like in the structure of the enzyme's binary complex with phosphate ion, instead of three, we observed two strong (closed) and four loose (open) conformations of the binding sites. We believe that closed active sites were filled with phosphate during the complex formation (prior to crystallization), and that sulphate ions from crystallization solutions $\left(1.1 \mathrm{~mol} \mathrm{dm}^{-3}\right)$ filled active sites in open conformation. As already mentioned, we can not distinguish between phosphate and sulphate ions in the electron density maps at given resolutions. Consequently, we can not exclude that phosphate ions bound to loose binding sites prior to crystallization were not replaced by sulphate ions abundantly present during crystallization. This structure supports our previous prediction that closed active sites correspond to strong binding of phosphate and open sites to its weak binding.

Recently we have prepared a series of ternary PNP complexes (not published yet) with phosphate and formycin A and in all of them we have found the same number and the same arrangement of closed and open active sites as in two just described binary complexes. Although available kinetic and binding studies are not in disagreement with this evidence, we have no explanation of why in one crystal structure three open and three closed active sites are present that regularly alternate ${ }^{13}$ while in all others to date available structures only two closed sites next to each other and four open sites exist.

\section{TERNARY COMPLEX OF $E$. coli PNP WITH FORMYCIN A AND PHOSPHATE - NEW PHOS- PHATE BINDING MODE}

After successful crystallization of the $E$. coli PNP binary complex (with phosphate) from crystallization conditions without sulphate ions, finally we have crystallized also one ternary complex (with phosphate and formycin A) from very similar solutions. ${ }^{6}$ This ternary complex was prepared with the aim to better understand the phosphorolysis, which is a two-substrate two-product reaction. As mentioned, formycins are substrate analogues and therefore can model substrate i.e. nucleoside binding. The complex crystallized in a hexagonal space group $P 6_{1} 22$ with only half of the hexamer in a asym- 


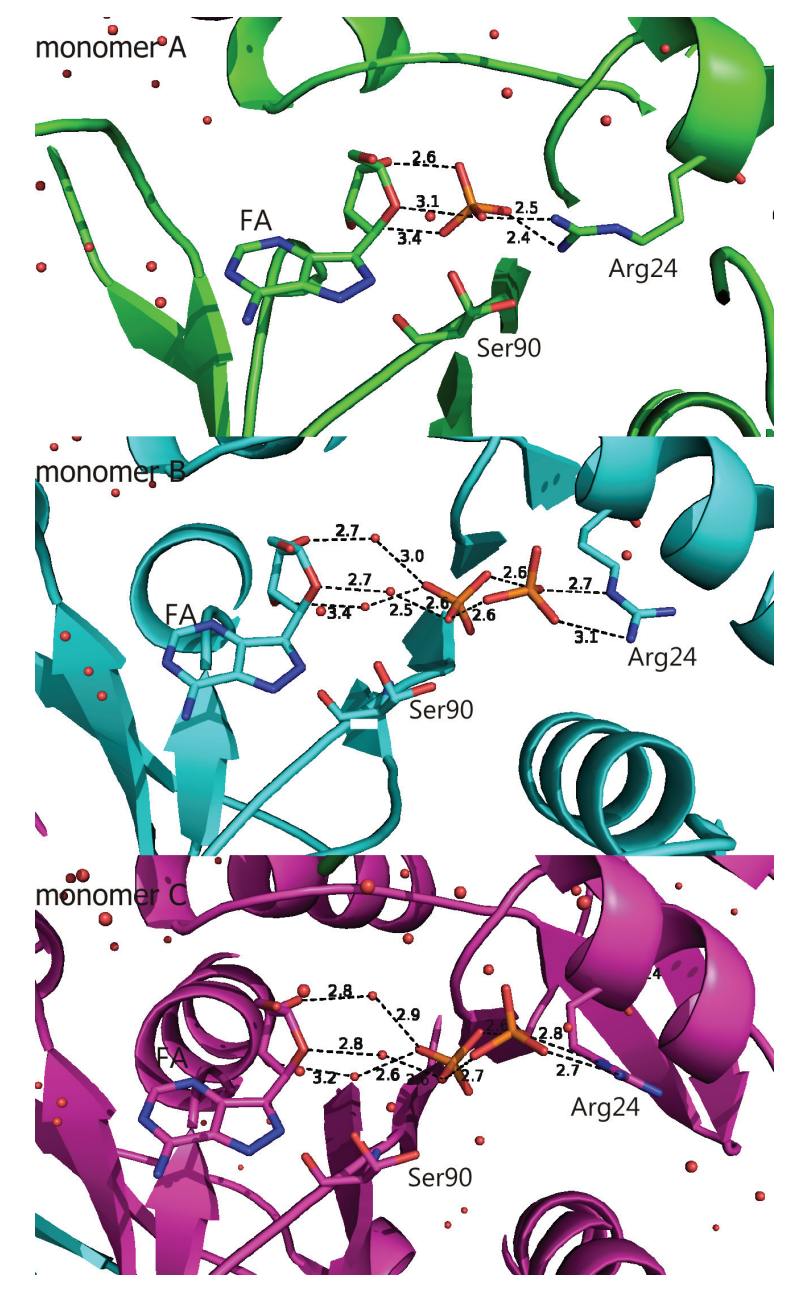

Figure 6. The comparison of the positions of phosphate molecules in three active sites of monomers A (closed conformation), $\mathrm{B}$ and $\mathrm{C}$ in the structure of $E$. coli PNP in complex with formycin $\mathrm{A}$ and phosphate. The three active sites are shown in approximately the same orientation.

metric unit (PDB code 3UT6). Monomers A and C form a dimer while monomer $\mathrm{B}$ forms a dimer with the symmetrically related monomer B'. Similarly as in many other PNP complex structures, two conformations of active sites were identified, open and closed. Two active sites in closed conformation are next to each other, but belong to neighbouring dimers, similarly as in the structure of binary complex with phosphate ions. All active sites are occupied by phosphate ions and formycin A (FA) molecules. The FA molecules are observed exactly in the same positions as in ternary complex crystallized with sulphate as precipitant. However, several new, to date not seen features were observed.

The active sites of monomers B and C (Figure 6) contain two phosphate molecules each. Interestingly, none of these ions make previously observed hydrogen bonds ${ }^{7,9,13,20,21}$ to the nucleoside and residues Gly20, Arg87, Ser90 and Arg43 from the neighbouring mono- mer. These two ions make hydrogen bonds to each other while the "standard" phosphate position is occupied by three water molecules. One of the two phosphates makes three hydrogen bonds with them. Water molecules are at hydrogen bond distance from pentose oxygen atoms of FA. The other phosphate ion is in close contact with two nitrogen atoms from the Arg24. The Arg24 side chain points away from the active site. Since the first E. coli PNP structure ${ }^{7}$ published in 1997 many PNP's complex structures have become available, but in none of them the above described configuration of phosphate ions had been found.

Active site of the monomer A is in close conformation with phosphate ion located at the "standard" position. It is hydrogen bonded with the pentose of the FA molecule, mimicking probable arrangement of two PNP natural substrates in the process of catalysis.

It is interesting to notice the differences between the only two PNP structures obtained from conditions not biased by the presence of sulphate: the binary complex with phosphate and the ternary complex with phosphate and FA. In the binary complex the stoichiometry is one phosphate per monomer and all phosphates are bound in their "standard" positions. In the ternary complex, which was obtained by soaking the binary complex in excess of FA, not only the stoichiometry (two phosphates per monomers whose active sites are in open conformations), but also the accommodation of phosphates in open active sites has changed. In this structure three water molecules occupy "standard" phosphate position and "shifted" two hydrogen-bonded phosphate ions more deeply in the binding pocket (Figure 6). Two active sites in closed conformation observed in each hexamer remained unchanged after binding of FA.

It is difficult to speculate if this situation is a consequence of very high phosphate concentration in crystallization, or very long crystallization period (one year), and if this structure has any relevance for the catalytic mechanism. To answer these questions new structures i.e. crystals grown in absence of phosphate and any of its analogues are needed.

\section{STOICHIOMETRY OF BINDING AND POSSIBLE COOPERATIVITY BETWEEN SUBUNITS OF PNP FROM STUDIES IN SOLUTION}

First indications pointing to the possible negative cooperativity between monomers forming the biologically active PNP form, both trimeric or hexameric (lowand high-molecular mass PNP families, respectively), come from kinetic studies (Figure 7).

The observed, so called "substrate activation" at high concentration of phosphate, and in some cases also of a nucleoside, i.e. deviation from linearity in 

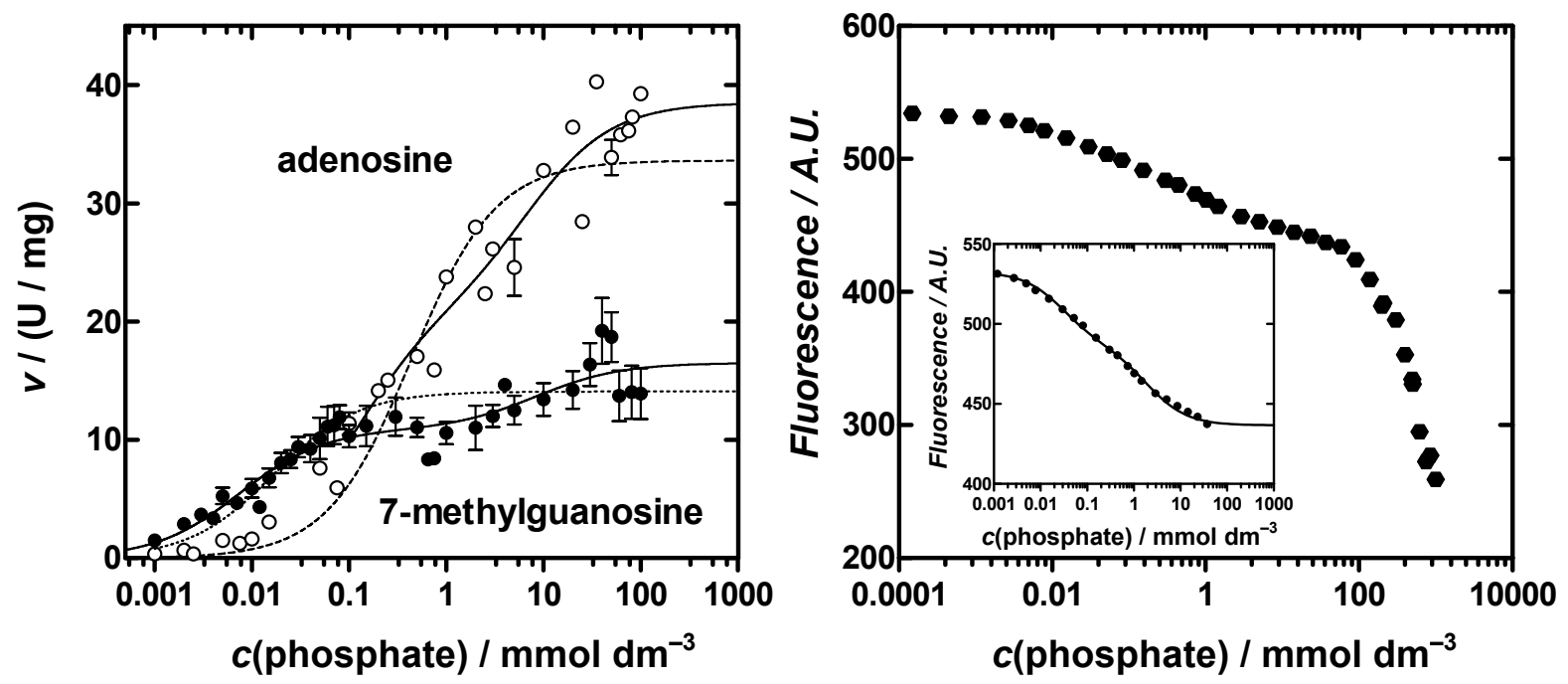

Figure 7. Left panel: kinetic properties of E. coli PNP with phosphate as variable substrate. Kinetics of phosphorolysis of the natural substrate, adenosine $\left(0.22 \mathrm{mmol} \mathrm{dm}{ }^{-3}\right)$ and the 7 -methylguanosine $\left(0.25 \mathrm{mmol} \mathrm{dm}{ }^{-3}\right)$ catalysed by the recombinant wild type E. coli PNP. Reactions were conducted at $298 \mathrm{~K}$ in $50 \mathrm{mmol} \mathrm{dm}{ }^{-3}$ Hepes buffer at $\mathrm{pH}=7.0$. Fitting was performed (solid lines) with the equation $v_{0}\left(c_{0}\right)=\left(2 V_{\max } c_{0} / K_{m 1}+2 b V_{\max 1} c_{0}^{2} /\left(a K_{m 1}^{2}\right)\right) /\left(1+2 c_{0} / K_{m 1}+c_{0}^{2} /\left(a K_{m 1}^{2}\right)\right)$, which takes into account cooperativity of binding sites. Two identical sites are characterised by constants $K_{\mathrm{m} 1}$ and $V_{\max }$, and allosteric interaction between these two sites leads to the change by factors $a$ and $b$, respectively, constants $K_{\mathrm{m} 1}$ and $V_{\max 1}$ of one site when the neighbouring site is occupied. ${ }^{18}$ Fitting of the Michaelis-Menten equation $v_{0}\left(c_{0}\right)=V_{\max 1} c_{0} /\left(c_{0}+K_{m 1}\right)$, although not appropriate to describe experimental data, is also shown for the comparison (dotted and dashed lines for adenosine and 7-methylguanosine, respectively). Right panel: fluorescence titrations of $E$. coli $\mathrm{PNP}$ with phosphate $298 \mathrm{~K}$ in $50 \mathrm{mmol} \mathrm{dm}{ }^{-3}$ Tris buffer at $\mathrm{pH}=7.6$. Equation $F([\mathrm{~L}])=F_{0}+\left(F_{0}-F_{\infty}\right)\left([\mathrm{L}] \mathrm{N}_{1} /\left([\mathrm{L}]+K_{\mathrm{d} 1}\right)+[\mathrm{L}]\left(1-\mathrm{N}_{1}\right) /\left([\mathrm{L}]+K_{\mathrm{d} 2}\right)\right)$, describing two kinds of binding sites with different dissociation constants, $K_{\mathrm{d} 1}, K_{\mathrm{d} 2}$, and relative binding stoichiometry $\mathrm{N}_{1}$ and $\left(1-\mathrm{N}_{1}\right)$, derived under assumption that protein concentration

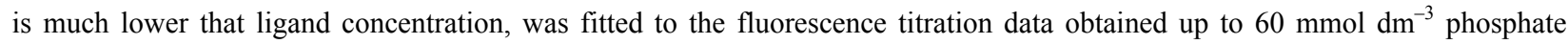
concentration (inset). For higher phosphate concentration fluorescence signal decreases, most probably due to increase of the ionic strength. $^{20}$

Lineweaver-Burk plot of $1 / v_{0}$ vs. $1 / c_{0}$ and in EdieHofstee plot of $v_{0} v s . v_{0} / c_{0}$, was interpreted as cooperative interactions between enzyme subunits. ${ }^{22-25}$ Binding studies of substrates and substrate analogues conducted with the use of fluorescence titrations $s^{11,13,18}$ and filtration methods ${ }^{26}$ led to conclusion that stoichiometry of binding may differ from one ligand molecule per enzyme subunit. However, these experiments yielded equivocal results. For example, in early studies by Jensen, 4 molecules of adenosine, and adenine in the presence of phosphate, but 7 molecules of ribose-1phosphate and 8 molecules of phosphate bound per $E$. coli enzyme hexamer were reported. ${ }^{26}$ However, these results should be treated with caution since calculations were based on the assumption of a Hill coefficient $n_{\mathrm{H}}=$ 0.5 and changing this parameter would also change the estimated binding stoichiometry. Koellner et al. ${ }^{13}$ and Kierdaszuk et al., ${ }^{18}$ reported 3 and 1 molecules of nucleoside ligand bound per $E$. coli enzyme hexamer, respectively, but only in the first report ${ }^{13}$ absolute concentration of active biding sites were obtained in the independent experiment (see discussion in the next paragraph). Binding of phosphate and sulphate (phos- phate analogue and inhibitor of PNP) by E. coli PNP, as mentioned above is best described by two dissociation constants $\left(29 \mu \mathrm{mol} \mathrm{dm}{ }^{-3}\right.$ and $\left.1.12 \mathrm{mmol} \mathrm{dm}^{-3}\right)$, and one dissociation constant $\left(1.36 \mathrm{mmol} \mathrm{dm}^{-3}\right)$, respectively, while total binding capacity for both anions was reported to be 6 molecules per enzyme hexamer. ${ }^{11}$

One of the possible reasons for such discrepancies observed may be the typical problem of all ligand binding studies; namely the proper determination of the absolute concentration of the binding sites. When the total protein concentration is used in calculations and protein is either not $100 \%$ pure or not fully active, lower stoichiometry of binding may be obtained. ${ }^{27}$ As shown by Koellner et al. ${ }^{13}$ stoichiometry of binding of nucleoside inhibitor (6-methyl-formycin A) by E. coli PNP is correlated with enzyme specific activity, so true stoichiometry may be obtained only when active sites concentrations are determined from total enzyme concentration corrected for the specific activity observed. However, even in such cases typical errors characteristic for stoichiometry studies are $\geq 15 \%$, which makes differentiation between stoichiometry e.g. 2 and 3 , or 3 and 4 , molecules per enzyme hexamer very problematic. 
Stoichiometry determination for phosphate, which is responsible for cooperativity between subunits, and non-identity of active sites ${ }^{20}$ (see below) is even more difficult since for two dissociation constants characterizing its binding, ${ }^{11} 29 \mu \mathrm{mol} \mathrm{dm}{ }^{-3}$ and $1.12 \mathrm{mmol} \mathrm{dm}^{-3}$, it is necessary to use comparable, hence very high, enzyme concentration to get absolute binding stoichiometry. Milimolar enzyme concentration will not be easy to obtain, although with overexpressed proteins it is in principle affordable, but is also outside of the useful range for some detection methods, like e.g. fluorescence titrations. Moreover, phosphate is an anion, so its addition to the solution in the course of titrations changes ionic strength of the medium, which may bias results of experiments with fluorescence detection. ${ }^{6}$

Recapitulating, stoichiometry of ligand binding by PNPs in solution, and possible cooperativity between subunits, or non-identity of active sites, call for more extensive studies. We have recently started such project with isothermal titration calorimetry (ITC) as a detection method, profiting from the very high overexpression yield of $E$. coli $\mathrm{PNP},{ }^{20,28}$ and with fluorescence detection using mutants of the $E$. coli enzyme with altered emission properties, but reliable unequivocal results are not yet available.

\section{CONCLUSION}

Despite years of effort, many accomplished research studies and scores of published scientific papers, the reaction mechanism of $E$. coli purine nucleoside phosphorylase still remains poorly understood. Actually, some recently published results instead of answering the existing open questions have opened new ones indicating even higher complexity of the problem than expected. New studies and new methods should be applied to understand allosteric regulation and cooperativity of phosphate and nucleoside binding to $E$. coli PNP.

Acknowledgements. This work was supported by the Ministry of Science, Education and Sports of the Republic of Croatia (Grant No. 098-1191344-2943) and Polish Ministry of Science and Higher Education (Grant No. N301044939).

\section{REFERENCES}

1. A. Bzowska, E. Kulikowska, and D. Shugar, Pharmacology \& Therapeutics 88 (2000) 349-425.

2. E. M. Bennett, R. Anand, P. W. Allan, A. E. Hassan, J. S. Hong, D. N. Levasseur, D. T. McPherson, W. B. Parker, J. A. Secrist 3rd, E. J. Sorscher, T. M. Townes, W. R. Waud, and S. E. Ealick, Chem. Biol. 10 (2003) 1173-1181.

3. Y. Zhang, W. B. Parker, E. J Sorscher, and S. E. Ealick, Curr. Top Med. Chem. 5 (2005) 1259-1274.

4. J. H. Zhou, B. Tang, X. L. Liu, D. W. He, and D. T. Yang, Chin. Med. J. 120 (2007) 1348-1352.

5. X. Xie, J. Guo, Y. Kong, G. X. Xie, L. Li, N. Lv, X. Xiao, J. Tang, X. Wang, P. Liu, M. Yang, Z. Xie, W. Wei, D. M. Spencer, and X. Xie, J. Gene. Med. 12 (2011) 680-691.

6. Z. Štefanić, M. Narczyk, G. Mikleušević, B. WielgusKutrowska, A. Bzowska, and M. Luić, FEBS Letters 586 (2012) 967-971.

7. C. Mao, W. J. Cook, M. Zhou, G. W. Koszalka, T. A. Krenitsky, and S. E. Ealick, Structure 5 (1997) 1373-1383.

8. M. S Hershfield, S. Chaffee, L. Koro-Johnson, A. Mary, A. A. Smith, and S. A. Short, Proc. Natl. Acad. Sci. U.S.A. 88 (1991) 7185-7189.

9. G. Koellner, M. Luić, D. Shugar, W. Saenger, and A. Bzowska, J. Mol. Biol. 280 (1998) 153-166.

10. M. Luić, G. Koellner, D. Shugar, W. Saenger, A. Bzowska, Acta Crystallogr. Sect. D 57 (2001) 30-36.

11. B. Kierdaszuk, A. Modrak-Wojcik, and D. Shugar, Biophys. Chem. 63 (1997) 107-118.

12. S. K. Burkley, and G. A. Petsko, Science 229 (1985) 23-28.

13. G. Koellner, A. Bzowska, B. Wielgus-Kutrowska, M. Luić, T. Steiner, W. Saenger, and J. Stępiński, J. Mol. Biol. 315 (2002) 351-371.

14. A. Bzowska, E. Kulikowska, and D. Shugar, Biochim. Biophys. Acta 1120 (1992) 239-247.

15. J. Wierzchowski and D. Shugar, Photochem. Photobiol. 35 (1982) 445-458.

16. P. C. Kline, and V. L. Schramm, Biochemistry 32 (1993) 13212 13219.

17. A. Bzowska, E. Kulikowska, and D. Shugar, Z. Naturforsch 48c (1993) 803-811.

18. B. Kierdaszuk, A. Modrak-Wojcik, J. Wierzchowski, and D. Shugar, Biochim. Biophys. Acta 1476 (2000) 109-128.

19. P. C. Kline, and V. L. Schramm, Biochemistry 34 (1995) 1153 1162 .

20. G. Mikleušević, Z. Štefanić, M. Narczyk, B. WielgusKutrowska, A. Bzowska, and M. Luić, Biochimie 93 (2011) 1610-1622.

21. E. M. Bennett, C. Li, P. W. Allan, W. B. Parker, and S. E. Ealick, J. Biol. Chem. 278 (2003) 47110-47118.

22. K. F. Jensen and P. Nygaard, Eur. J. Biochem. 51 (1975) 253 265.

23. J. D. Stoeckler, Purine nucleoside phosphorylase: a target for chemotherapy, in: R. J. Glazer (Eds.), Developments in Cancer Chemotherapy, CRC Press, Boca Raton, 1984, pp. 35-60.

24. A. Ropp, and T. W. Traut, J Biol Chem 266 (1991) 7682-7687.

25. B. Wielgus-Kutrowska, J. Tebbe, W. Schroeder, M. Luić, D. Shugar, W. Saenger, G. Koellner, and A. Bzowska, Adv. Exp. Med. Biol. 431 (1998) 259-264.

26. K. F. Jensen, Eur. J. Biochem. 67 (1976) 377-386.

27. A. Fersht, The absolute concentration of enzyme, in: Structure and mechanism in protein science, W.H. Freeman and Company, New York, 1999, pp. 155-158.

28. J. Lee, S. Filosa, J. Bonvin, S. Guyon, R. A. Aponte, and J. L. Turnbull, Prot. Exp. Pur. 22 (2001) 180-188. 